\title{
Physicochemical characterization and dissolution studies of acyclovir solid dispersions with Pluronic F127 prepared by the kneading method
}

\author{
BOŻENA KAROLEWICZ ${ }^{1 *}$ \\ KAROL NARTOWSKI ${ }^{1,2}$ \\ JANUSZ PLUTA ${ }^{1}$ \\ AGATA GÓRNIAK ${ }^{3}$ \\ ${ }^{1}$ Department of Drug Form Technology \\ Faculty of Pharmacy, Wroclaw Medical \\ University, Wroclaw 50556, Poland \\ ${ }^{2}$ Current address: School of Pharmacy \\ University of East Anglia, Norwich \\ Research Park, Norwich, Norwich \\ NR4 7TJ, UK \\ ${ }^{3}$ Laboratory of Elemental Analysis \\ and Structural Research, Faculty of \\ Pharmacy, Wroclaw Medical \\ University, Wroclaw 50556, Poland \\ Accepted September 28, 2015 \\ Online published October 27, 2015
}

\begin{abstract}
The dissolution rate of anhydrous acyclovir was improved by the preparation of physical mixtures and solid dispersions with the non-ionic polymer Pluronic F127 using the kneading method at different drug-to-polymer ratios. The obtained physical mixtures and solid dispersions were examined in terms of drug content and possible physical and chemical interactions between the drug and polymer using FTIR spectral studies, differential scanning calorimetry and powder X-ray diffraction analysis. The dissolution rate of acyclovir was determined using the rotating disk method. It was found that the minimal content of the polymer within the mixtures needed to increase the dissolution rate of the drug was $50 \%$.
\end{abstract}

Keywords: acyclovir, Pluronic F127, solid dispersions, physical mixtures, kneading, dissolution improvement

Acyclovir (ACV) is an antiviral compound used in the treatment of diseases caused by the Herpes simplex (type 1) and Varicella zoster viruses $(1,2)$. Administration of acyclovir via the oral route leads to low and variable bioavailability (15-30\%) (3). This classifies ACV as a BCS class III or IV drug with low permeability and high solubility or very low solubility and permeability (4). Furthermore, multiple administration of large doses of the drug required to achieve therapeutic concentration in the blood increases possible side effects such as nausea, diarrhoea and headaches, resulting in patient non-compliance (5).

ACV forms two hydrates with 3:2 (commercial ACV, form V) and 1:2 (form VI) drug-to-water ratios and four anhydrous crystalline forms: two stable under ambient conditions (forms I and II) and two metastable high temperature forms (forms III and IV) $(6,7)$. In this work, we use the nomenclature of ACV forms as proposed by Lutker et al. (6). The anhydrous form I dissolves relatively slower than the hydrated form V (8). Active ingredients existing in different crystalline forms may affect absorption of the drug from oral dosage

\footnotetext{
*Correspondence; e-mail: bozena.karolewicz@umed.wroc.pl
} 
forms (9). Various methods for increasing dissolution of ACV have been described, including micronization, nanosuspension, solid dispersions (10), inclusion complex with cyclodextrin (11), co-crystallisation, amorphisation (12) or formulation of microemulsions (10, 13) and niosomes $(5,14,15)$. Dissolution rates of ACV from solid dispersions with urea, mannitol and selected polymers were compared to the pure substance $(14,16,17)$. In studies done by Kushwaha et al. $(14,16)$ the plasma concentration of ACV in rabbits, after a single dose of a pure substance as a solution and as a solid dispersion, showed significantly higher $A U C$ values (5 times) obtained from the latter.

It was recently shown by our group and other authors that Pluronic F127 can be used successfully as a carrier for dissolution rate improvement of poorly water-soluble drugs, including simvastatin (18) and ketoconazole (19-22). The aim of this study was to increase acyclovir dissolution rate by preparing physical mixtures and solid dispersions of anhydrous acyclovir form I (ACV) with Pluronic F127 (PLU).

\section{EXPERIMENTAL}

\section{Materials}

The acyclovir used in the study was a gift from Valeant Pharmaceuticals International, Inc., Poland. Pluronic F127 was purchased from Fluka Biochemica (Germany). Sodium lauryl sulfate (SLS) was purchased from P. P. H. Stanlab (Poland). The HPLC grade methanol was obtained from Chempur (Poland). Finally, potassium bromide and hydrochloric acid $37 \%$ were obtained from POCH (Poland). All other chemicals and reagents were of analytical grade.

\section{Methods}

Preparation of anhydrous form of acyclovir (form I). - Commercially available acyclovir (polymorphic form V of ACV) is a 3:2 ACV:water hydrate (6). The anhydrous crystalline form of acyclovir (form I) was prepared according to the method described by Lutker et al. (6). Ten grams of ACV form $\mathrm{V}$ was briefly dehydrated by heating in an oven at a temperature range from 25 to $180{ }^{\circ} \mathrm{C}$ using a heating rate of $5{ }^{\circ} \mathrm{C} \mathrm{min}{ }^{-1}$. After cooling, the samples were stored in desiccators at room temperature until use. The formation of anhydrous crystalline form I of acyclovir was confirmed by DSC, XRPD and FTIR and it was used to formulate physical mixtures and solid dispersions.

Preparation of physical mixtures (PM). - ACV and PLU were weighed accurately and mixed in an agate mortar for 10 minutes in order to obtain a homogenous mixture. The mass ratios of the mixtures were: 90/10, 80/20, 70/30, 60/40, 50/50, 40/60, 30/70, 20/80 and $10 / 90 \%$. The obtained solid dispersions were sieved through a $315-\mu \mathrm{m}$ sieve and stored in a desiccator (at room temperature) until further analysis.

Preparation of solid dispersions using the kneading method (KN). - Mixtures of ACV and PLU were prepared by grinding accurately weighed quantities of each compound in an agate mortar with $2 \mathrm{~mL}$ of $97 \%$ methanol for 10 minutes. After solvent evaporation, dry powder was again triturated in an agate mortar and sieved through a sieve with a mesh size of $315 \mu \mathrm{m}$. The mass ratios of the mixtures were: 90/10, 80/20, 70/30, 60/40, 50/50, 40/60, $30 / 70,20 / 80$, and 10/90. The obtained solid dispersions were stored in a desiccator at room temperature until use. 
Drug content determination. - Ten-mg equivalent of anhydrous ACV in either physical mixture or solid dispersion was dissolved in $100 \mathrm{~mL}$ of $0.1 \mathrm{~mol} \mathrm{~L}^{-1} \mathrm{HCl}$. The prepared solutions were diluted in a 1:10 ratio and the drug was determined spectrophotometrically at $252 \mathrm{~nm}$ using a UV-Vis spectrophotometer (Jasco V-650, Japan). The percentage of drug loading was calculated for all mixtures.

Differential scanning calorimetry (DSC). - The DSC measurement of each mixture was performed using a differential scanning calorimeter (Mettler Toledo DSC 25, Switzerland) equipped with a heat-flow sensor and connected via an interface TA Controller TC 15 to a computer. Measurements were taken by STAR ${ }^{\mathrm{e}}$ software. Samples for DSC measurements were sealed in $40-\mu \mathrm{L}$ standard aluminum crucibles with a single hole punched in the lid. An empty pan of the same type was employed as reference. The DSC instrument was calibrated using the melting point of indium $\left(156.6 \pm 0.3^{\circ} \mathrm{C}\right)$ as a standard. DSC scans of each sample were performed at a heating rate of $5^{\circ} \mathrm{C} \mathrm{min}^{-1}$ in a temperature range of 25 to $300{ }^{\circ} \mathrm{C}$. The DSC cell was purged with a stream of dry argon at a rate of $50 \mathrm{~mL} \mathrm{~min}^{-1}$.

Powder X-ray diffraction analysis (XRPD). - Powder X-ray diffraction patterns of anhydrous ACV, PLU, their physical mixtures and solid dispersions were recorded at room temperature using an Ultima IV powder diffractometer, equipped with a Cross Beam Optics (Rigaku, Japan) with CuK $\alpha$ radiation. The scanning rate of $10^{\circ} \mathrm{min}^{-1}$ was applied between 3 and $60^{\circ}(2 \theta)$.

Fourier transform infrared spectroscopy (FTIR). - FTIR spectra were acquired using an IR Affinity-1 spectrometer (Shimadzu, Japan) from 4000 to $200 \mathrm{~cm}^{-1}$. Prior to analysis, samples were mixed in an agate mortar with $\mathrm{KBr}$ powder (spectroscopic grade) and were compressed into a 13-mm disc under the pressure of 10 tons for 1 minute using a hydraulic press (Specac Ltd., UK). The KBr spectrum was used as background.

Dissolution studies. - Dissolution studies were carried out for anhydrous ACV, physical mixtures and solid dispersions at all drug-to-polymer ratios. The dissolution system was fitted with an SR8-PLUS dissolution bath (Hanson, USA) and a 7-channel peristaltic pump. The dissolution rate was measured using the pharmacopeial rotating disk method (25). ACV (100.0 mg), or an equivalent amount of solid dispersion, was prepared by compressing powder into discs using a hydraulic press (Specac Ltd.) for 1 minute under a compression force of $1 \mathrm{t}$ using an 8 -mm punch. The die was mounted on the stirring drive mechanism and was rotated at $50 \mathrm{rpm}$. The dissolution test was conducted under sink conditions in $1000 \mathrm{~mL}$ of distilled water containing $0.5 \%$ SLS at $37 \pm 0.5^{\circ} \mathrm{C}$. Samples were collected after 5, 10, 15 20, 25, 30, 45 and 60 min of the study. The ACV quantity was determined spectrophotometrically at $252 \mathrm{~nm}$. The calibration curve was constructed by measuring ACV absorbance in $0.5 \%$ SLS solution ranging from 1.6 to $8.0 \mu \mathrm{g} \mathrm{mL}^{-1}\left(R^{2}=0.999\right)$.

\section{RESULTS AND DISCUSION}

\section{Drug in physical mixtures and solid dispersions}

It is of paramount importance in pharmaceutical drug delivery to ensure a uniform content of API within the formulations as well as monitoring of possible phase transitions of the drug during processing. Acyclovir content within the formulated physical mixtures and solid dispersions, in the range of 97.6 to $101.7 \%$ of the weighed amount, was in good agreement with expected values. As several solvates and polymorphic forms of ACV had 
been reported in the literature, careful control over formulation processes and monitoring of the final phase of the active pharmaceutical ingredient (API) within the physical mixtures and solid dispersions was investigated using DSC, PXRD and FTIR (6-9).

The DSC thermogram of ACV form I (Fig. 1a) shows three thermal effects. The first endotherm can be assigned to reversible phase transition of ACV form I to ACV form IV at $172.9^{\circ} \mathrm{C}$, followed by melting of ACV form IV at $255.1^{\circ} \mathrm{C}$ and exothermic decomposition at $258.7^{\circ} \mathrm{C}$. Incorporation of $\mathrm{ACV}$ in the polymer matrix does not affect the onset of the first phase transition of ACV form I to ACV form IV in all investigated drug-to-polymer ratios of both physical mixtures and solid dispersions (Figs. 1b and c). The magnitude of heat (corresponding to the enthalpy of solid-solid phase transition) increases gradually with the increasing ACV mass fraction. The second peak, corresponding to the melting of the stable anhydrous $\mathrm{ACV}$ form IV at $255.1^{\circ} \mathrm{C}$, was generally broader, indicating that complete melting took place over a wider temperature range. The broadening of ACV melting peak

a)

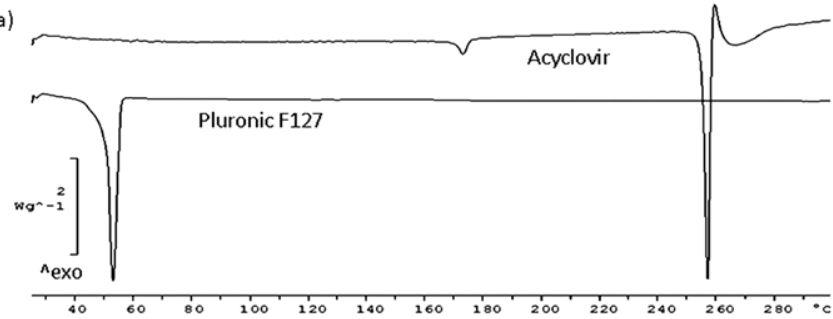

b)

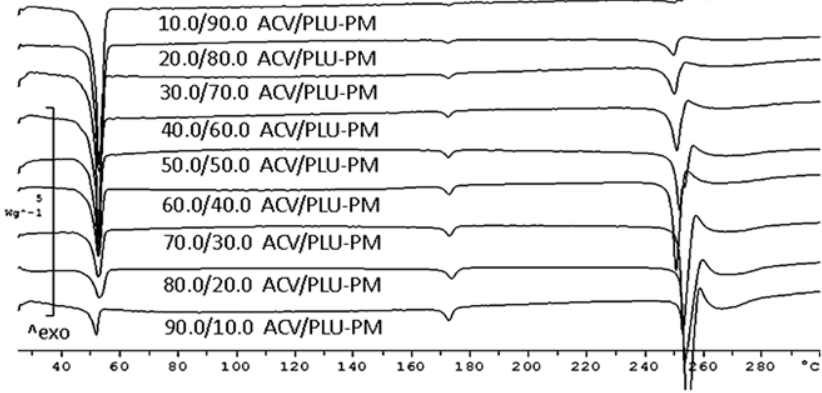

c)

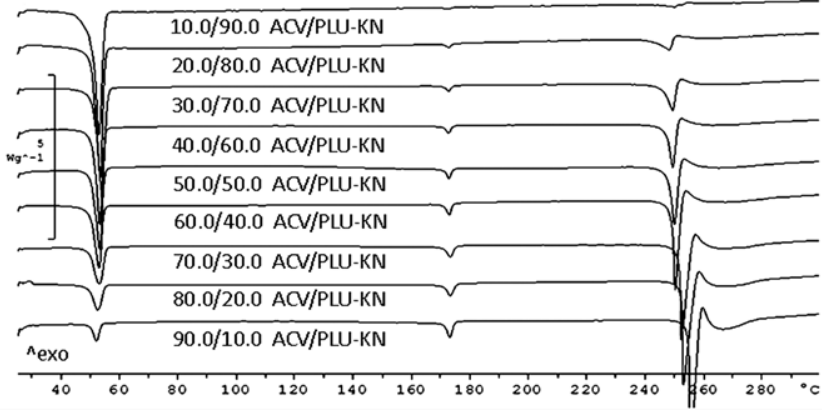

Fig. 1. DSC thermograms of: a) anhydrous ACV and PLU, b) ACV/PLU physical mixtures (PM) and c) ACV/PLU solid dispersions obtained by the kneading method (KN). 
results from the increase in the polymer content in physical mixtures or solid dispersions. The DSC curve of PLU (Fig. 1a) shows only one endothermic event, associated with its melting at $52.6^{\circ} \mathrm{C}$. The DSC thermograms of the studied physical mixtures and solid dispersions show only phase transitions of PLU and ACV form I, indicating that mixing of both compounds does not affect the polymorphic order of ACV and the phase transition of either compound.

The DSC data are further corroborated by XRPD and FTIR analysis. The diffraction pattern of ACV used to prepare the formulations can be assigned to the anhydrous ACV form I based on characteristic peaks at 8.52, 11.66, 24.07 and $28.74^{\circ} 2 \theta$. ACV form I can be obtained by slow dehydration of the ACV form $\mathrm{V}$, which recrystallizes into monoclinic $\mathrm{P} 2_{1} / \mathrm{c}$ space group with $\mathrm{Z}^{\prime}=1$, as reported by Lutker et al. (6). Due to the reversible phase transition between ACV form I and ACV form V at elevated humidity, it is important to assure phase stability during mixing with the polymer. XRPD diffraction patterns of the investigated physical mixtures and solid dispersions show characteristic patterns of ACV form I, indicating stability of the crystalline phase after formulation with PLU. Furthermore, two distinct peaks of semicrystalline PLU, at 19.12 and $23.26^{\circ} 2 \theta$, can be observed. All XRPD patterns of physical mixtures and solid dispersions show superposition of both ACV form I and PLU peaks, indicating no changes in the crystalline phase and crystallinity of either material.

Differences in the dissolution rate of physical mixtures or solid dispersions may result from intermolecular interactions in the solid state between API and polymer (24). Infrared spectroscopy is highly sensitive probe to investigate inter- and intra-molecular interactions in the solid state. Furthermore, IR enables to distinguish between the drug polymorphic forms due to its sensitivity to structural differences arising from distinct hydrogen bonding patterns within different crystalline forms (25). The FTIR spectrum of anhydrous ACV form I shows a characteristic N-H vibration region with two peaks at 3433.29 and $3398.57 \mathrm{~cm}^{-1}$. The peaks for primary amines are present at 3280.92 and $3180.62 \mathrm{~cm}^{-1}$ and the peak characteristic of the secondary amines is clearly visible at $3088.03 \mathrm{~cm}^{-1}$. Furthermore, the sharp peak at $1629.85 \mathrm{~cm}^{-1}$ represents an $\mathrm{N}-\mathrm{H}$ bond of primary amines and the peak

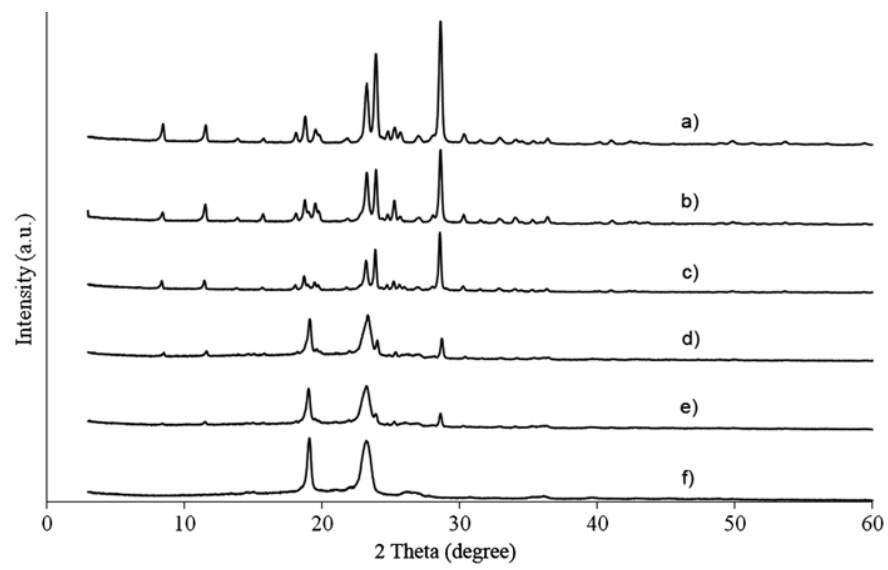

Fig. 2. XRPD patterns of: a) anhydrous ACV, b) 80/20 (\%, $\mathrm{m} / \mathrm{m})$ ACV/PLU-PM, c) 80/20 (\%, $\mathrm{m} / \mathrm{m})$ ACV/ PLU-KN, d) 20/80 (\%, $m / m)$ ACV/PLU-PM, e) 20/80 (\%, $\mathrm{m} / \mathrm{m})$ ACV/PLU-KN, f) PLU. 
B. Karolewicz et al:: Physicochemical characterization and dissolution studies of acyclovir solid dispersions with Pluronic F127 prepared by the kneading method, Acta Pharm. 66 (2016) 119-128.

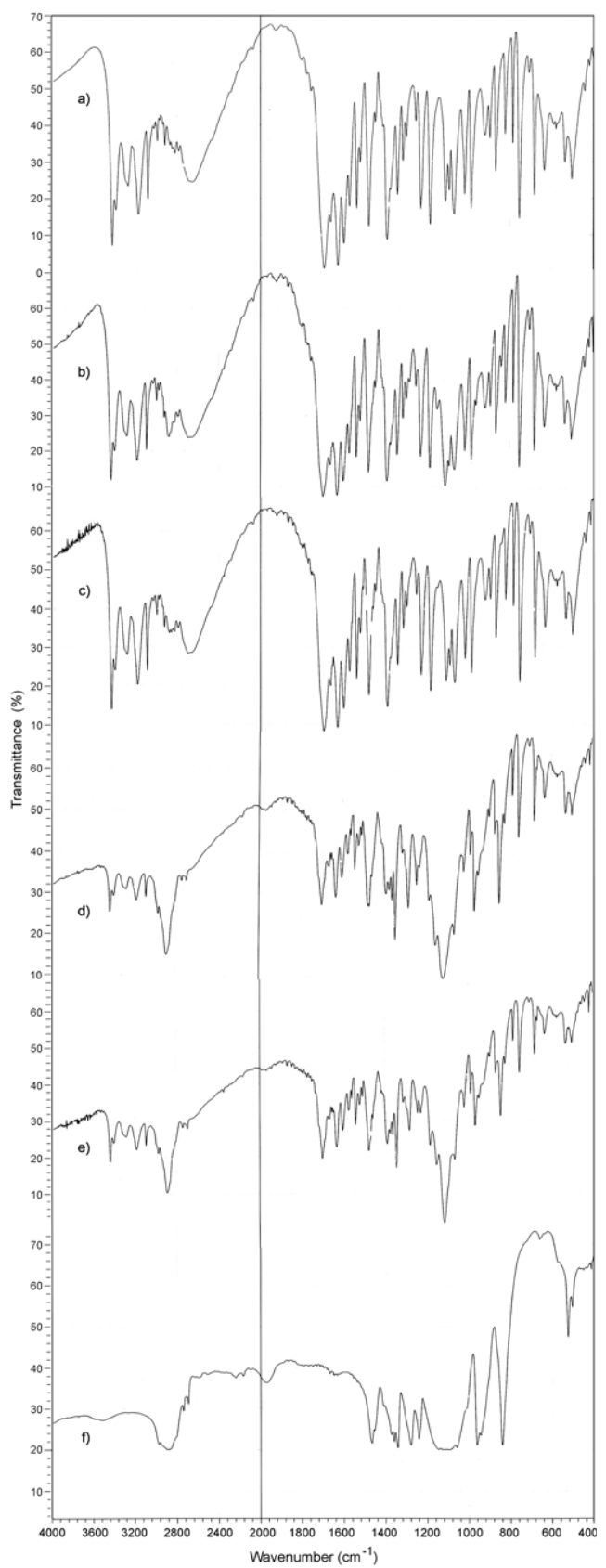

Fig. 3. FTIR spectra of: a) anhydrous ACV, f) PLU, and selected solid dispersions: b) 80/20 (\%, $\mathrm{m} / \mathrm{m})$ ACV/PLU-PM, c) 20/80 (\%, $m / m)$ ACV/PLU-KN, d) 20/80 (\%, $m / m)$ ACV/PLU-PM, e) 20/80 (\%, $m / m)$ ACV/ PLU-KN. 
visible at $1695.43 \mathrm{~cm}^{-1}$, the stretching vibration of the $-\mathrm{C}=\mathrm{O}$ group of API. The occurrence of a combination of band patterns in the wavenumber region of $2000-1650 \mathrm{~cm}^{-1}$ and the $\mathrm{CH}$-stretch region of the FTIR spectrum above $3000 \mathrm{~cm}^{-1}$ indicates the presence of an aromatic group. The spectrum of Pluronic F127 shows two characteristic absorption peaks at 1242.16 and $1149.57 \mathrm{~cm}^{-1}$ arising from C-O stretching vibrations (Fig. 3). However, both drug and polymer may potentially form multiple hydrogen bonds due to the presence of amine and hydroxyl groups in ACV and carbonyl groups in PLU. FTIR spectra of the physical mixtures and solid dispersions are superpositions of the spectra of individual components. Such results show no interactions between the components after formulation and indicate chemical stability of the drug and the mixtures obtained after processing.

\section{Dissolution studies}

The intrinsic dissolution rate (IDR) is of great interest in preformulation research as it enables, better correlation of in vitro results with in vivo behavior of the drug (26). IDR is defined as the dissolution rate of a pure compound under the condition of constant surface area. IDR was determined according to the equation (27):

IDR $=\left(\frac{\mathrm{d} m}{\mathrm{~d} m}\right)_{\max } / A$

where $(\mathrm{d} t)_{\max }$ is the maximum slope in the dissolution curve evaluated at the start of the dissolution process, $A$ is the area of the drug disk $\left(\mathrm{cm}^{2}\right), m$ is the mass $(\mathrm{mg}), t$ is the time (min). As determination of the dissolution rate in comparison with solubility studies does not depend on saturation concentration of API in the medium, it is less sensitive to the errors related to possible phase changes within the formulation. Furthermore, physical changes of API at the surface exposed to the dissolution medium, including recrystallization from the amorphous state or changes in hydration levels, can be easily monitored by rapid changes in the slope of the dissolution curve (26-29). Fig. 4 shows the amount of anhydrous ACV dissolved from physical mixtures and solid dispersions with PLU in a 0.5 $\%$ SLS solution as a function of time. Results suggest that the dissolution rate of ACV form I from physical mixtures and solid dispersions containing more than $60 \%(\mathrm{~m} / \mathrm{m})$ of PLU is higher compared to that of anhydrous ACV form I. After 60 minutes of the test, over $26 \%$ of ACV was released from the solid dispersion 10/90\% $(\mathrm{m} / \mathrm{m}) \mathrm{ACV} / \mathrm{PLU}$ prepared by the kneading method, while at that time only $11 \%$ of pure ACV had dissolved. A decrease in dissolution was observed for physical mixtures and solid dispersions containing $60-90 \%$ $(\mathrm{m} / \mathrm{m})$ of ACV. As the polymer concentration increases, dissolution also increases due to enhanced disaggregation and wetting of ACV particles in the presence of the hydrophilic group of the polymer and surface active properties of PLU (21). In the dissolution medium, the hydrophilic polymer undergoes rapid dissolution, maintaining a high concentration in the diffusion layer of the drug and thus improving the dissolution of the drug in this layer and thereby increasing the dissolution rate of the substance. Furthermore, formation of PLU micelles in dissolution medium may also lead to increased dissolution of ACV, since it can be partly entrapped within the hydrophobic core of the micelles (30). IDR of anhydrous ACV, its physical mixtures and solid dispersions with PLU and the linear relationship between the amount of the dissolved drug and time are given in Table I. These results further indicate no phase transformation of ACV form I to its hydrated form (ACV form $\mathrm{V}$ ) within the time of the dissolution studies, since there is no change in the slope of the dissolution curve determined by almost perfect fit $\left(R^{2}=0.99\right)$ to the linear function. 
B. Karolewicz et al:: Physicochemical characterization and dissolution studies of acyclovir solid dispersions with Pluronic F127 prepared by the kneading method, Acta Pharm. 66 (2016) 119-128.
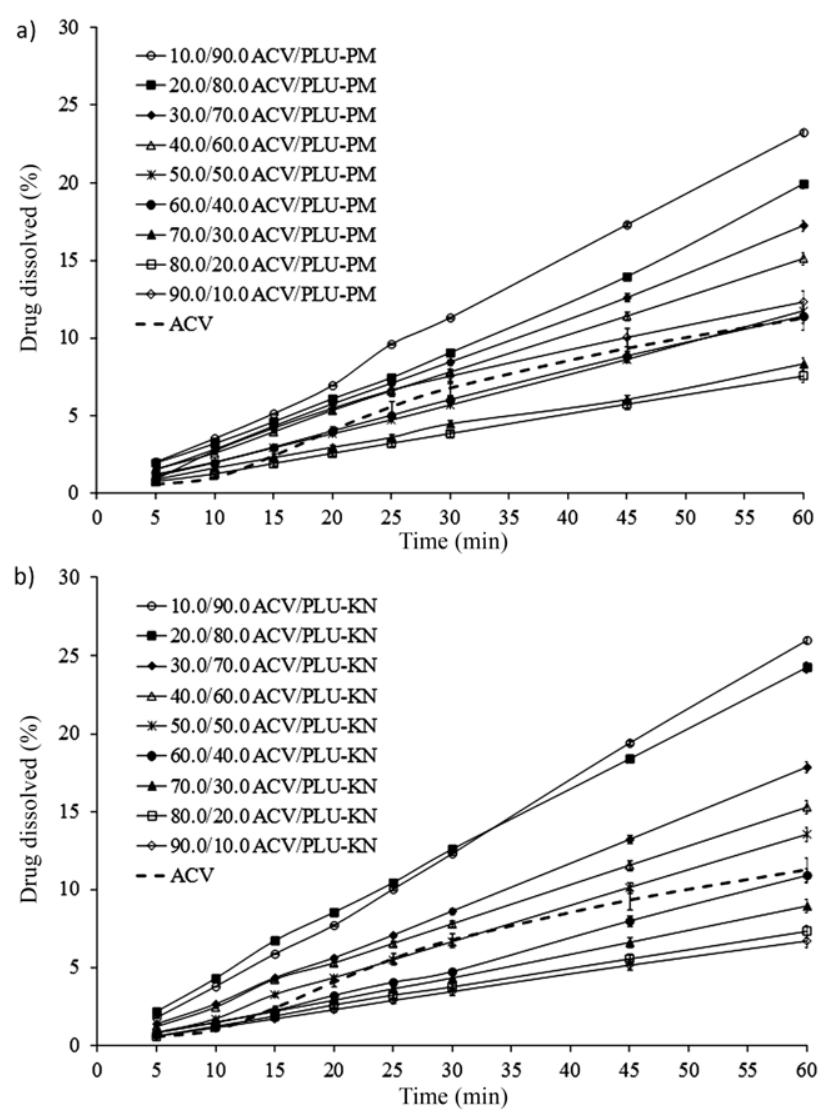

Fig. 4. The dissolution profiles of: a) physical mixtures and b) solid dispersions in $0.5 \%$ sodium lauryl sulfate aqueous solution.

Table I. IDR of ACV and its mixtures, solid dispersions with PLU and the corresponding ratios

\begin{tabular}{lccc}
\hline Formulation & IDR $\left(\mathrm{mg} \mathrm{cm}^{-2} \mathrm{~min}^{-1}\right)$ & $R^{2}$ & IDR ratio formulation/ACV \\
\hline $10 / 90(\%, m / m)$ ACV/PLUPM & $0.9534 \pm 0.0005$ & 0.9693 & 2.30 \\
$20 / 80(\%, m / m)$ ACV/PLUPM & $0.6502 \pm 0.0033$ & 0.9943 & 1.57 \\
$30 / 70(\%, m / m)$ ACV/PLUPM & $0.5699 \pm 0.0021$ & 0.9995 & 1.38 \\
$40 / 60(\%, m / m)$ ACV/PLUPM & $0.4976 \pm 0.0135$ & 0.9995 & 1.20 \\
$10 / 90(\%, m / m)$ ACV/PLUKN & $0.8883 \pm 0.0014$ & 0.9989 & 2.14 \\
$20 / 80(\%, m / m)$ ACV/PLUKN & $0.7995 \pm 0.0023$ & 0.9993 & 1.93 \\
$30 / 70(\%, m / m)$ ACV/PLUKN & $0.6006 \pm 0.0067$ & 0.9995 & 1.45 \\
$40 / 60(\%, m / m)$ ACV/PLUKN & $0.5100 \pm 0.0325$ & 0.9987 & 1.23 \\
ACV & $0.4144 \pm 0.0623$ & 0.9733 & - \\
\hline
\end{tabular}

Mean $\pm \operatorname{SD}(n=3)$. 
B. Karolewicz et al.: Physicochemical characterization and dissolution studies of acyclovir solid dispersions with Pluronic F127 prepared by the kneading method, Acta Pharm. 66 (2016) 119-128.

\section{CONCLUSIONS}

In this paper, we have demonstrated the application and evaluation of the non-ionic polymer Pluronic F127 for the preparation of physical mixtures and solid dispersions with acyclovir. It was found that the minimal content of the polymer within the mixtures needed to increase the dissolution rate of the drug is $50 \%$. The phase of ACV form I did not change during the preparation of physical mixtures or solid dispersions, which was proven by DSC, FTIR and XRPD. This indicates successful incorporation of ACV form I within the polymer matrix without polymorphic transitions of API.

Acknowledgements. - The authors thank Valeant Pharmaceuticals International, Inc. Poland for providing the acyclovir used in this study.

\section{REFERENCES}

1. C. Cernik, K. Gallina and R. T. Brodell, The treatment of herpes simplex infections: an evidencebased review, Arch. Intern. Med. 168 (2008) 1137-1144; DOI: 10.1001/archinte.168.11.1137.

2. K. S. Erlich, Management of herpes simplex and varicella-zoster virus infections, West. J. Med. 166 (1997) 211-215.

3. D. M. Thappa, Textbook of Dermatology, Leprology, and Venereology, 3rd ed., Elsevier, Gurgaon 2009, pp. 350-351.

4. J. Arnal, I. Gonzalez-Alvarez, M. Bermejo, G. L. Amidon, H. E. Junginger, S. Kopp, K. K. Midha, V. P. Shah, S. Stavchansky, J. B. Dressman and D. M. Barends, Biowaiver monographs for immediate release solid oral dosage forms: acyclovir, J. Pharm. Sci. 97 (2008) 5061-5073; DOI: 10.1002/jps. 21392.

5. R. Sankar and S. Jain, Approaches for enhancing the bioavailability of acyclovir: a critical review, Int. J. Pharm. Biol. Sci. 4 (2013) 623-633.

6. K. M. Lutker, R. Quinones, J. Xu, A. Ramamoorthy and A. J. Matzger, Polymorphs and hydrates of acyclovir, J. Pharm. Sci. 3 (2011) 949-963; DOI: 10.1002/jps.22336.

7. K. Terada, H. Kurobe, M. Ito, Y. Yoshihashi, E. Yonemochi, K. Fujii and H. Uekusa, Polymorphic transformation behavior of acyclovir based on the thermodynamics and crystallography, J. Therm. Anal. Calorim. 113 (2013) 1261-1267; DOI: 10.1007/s10973-013-3140-1.

8. A. Kristl, S. Srcic, F. Vrecer, B. Sustar and D. Vojnovic, Polymorphism and pseudopolymorphism: influencing the dissolution properties of the guanine derivative acyclovir, Int. J. Pharm. 139 (1996) 231-235; DOI: 10.1016/0378-5173(96)04601-7.

9. Y. T. Sohn and S. H. Kim, Polymorphism and pseudopolymorphism of acyclovir, Arch. Pharm. Res. 31 (2008) 231-234; DOI: 10.1007/s12272-001-1146-x.

10. P. K. Ghosh, R. J. Majithiya, M. L. Umrethia and R. S. R. Murthy, Design and development of microemulsion drug delivery system of acyclovir for improvement of oral bioavailability, AAPS PharmSciTech. 7 (2006) 172-177; DOI: 10.1208/pt070377.

11. C. Von Plessing Rossel, J. S. Carreño, M. Rodríguez-Baeza and J. B. Alderete, Inclusion complex of the antiviral drug acyclovir with cyclodextrin in aqueous solution and in solid phase, Quimica Nova 23 (2000) 749-752; DOI: 10.1590/S0100-40422000000600007.

12. T. Masuda, Y. Yoshihashi, E. Yonemochi, K. Fujii, H. Uekusa and K. Terada, Cocrystallization and amorphization induced by drug-excipient interaction improves the physical properties of acyclovir, Int. J. Pharm. 422 (2012) 160-169; DOI: 10.1016/j.ijpharm.2011.10.046. 
B. Karolewicz et al.: Physicochemical characterization and dissolution studies of acyclovir solid dispersions with Pluronic F127 prepared by the kneading method, Acta Pharm. 66 (2016) 119-128.

13. D. Patel and K. K. Sawant, Oral bioavailability enhancement of acyclovir by self-microemulsifying drug delivery systems (SMEDDS), Drug Dev. Ind. Pharm. 33 (2007) 1318-1326; DOI: 10.1080/ 03639040701385527.

14. A. Kushwaha, S. K. Prajapati and B. Sharma, Comparative study of acyclovir solid dispersion for bioavailability enhancement, AJPTR 1 (2011) 179-201.

15. R. Cortesi and E. Esposito, Acyclovir delivery systems, Expert Opin. Drug Deliv. 5 (2008) 1217-1230; DOI: $10.1517 / 17425240802450340$.

16. A. Kushwaha, Solid dispersion: an approach to enhance the dissolution rate of acyclovir, Int. J. Nov. Drug Deliv. Tech. 2 (2012) 291-296.

17. N. Sachan, S. Pushkar, S. S. Solanki and D. S. Bhatere, Enhancement of solubility of acyclovir by solid dispersion and inclusion complexation methods, World Appl. Sci. J. 11 (2010) 857-864.

18. B. Karolewicz, M. Gajda, A. Owczarek, J. Pluta and A. Górniak, Physicochemical and dissolution studies of simvastatin solid dispersions with Pluronic F127, Pharmazie 69 (2014) 589-594; DOI: 10. 1691/ph.2014.3217.

19. B. Karolewicz, A. Górniak, A. Owczarek, E. Żurawska-Płaksej, A. Piwowar and J. Pluta, Thermal, spectroscopic, and dissolution studies of ketoconazole-Pluronic F127 system, J. Therm. Anal. Calorim. 115 (2014) 2487-2493; DOI: 10.1007/s10973-014-3661-2.

20. G. A. Shazly, M. A. Ibrahim, M. M. Badran and K. M. A. Zoheir, Utilizing Pluronic F-127 and Gelucire 50/13 solid dispersions for enhanced skin delivery of flufenamic acid, Drug Dev. Res. 73 (2012) 299-307; DOI: 10.1002/ddr.21013.

21. P. Kumar, C. Mohan, M. K. S. U. Shankar and M. Gulati, Physiochemical characterization and release rate studies of solid dispersions of ketoconazole with Pluronic F127 and PVP K-30, Iran. J. Pharm. Res. 10 (2011) 685-694.

22. N. Kolašinac, K. Kachrimanis, I. Homšek, B. Grujič, Z. Đurič and S. Ibrič, Solubility enhancement of desloratadine by solid dispersion in poloxamers, Int. J. Pharm. 15 (2012) 161-170; DOI: 10.1016/j. ijpharm.2012.06.060.

23. European Pharmacopoeia, 8th ed., European Directorate for the Quality of Medicines \& Healthcare, Strasbourg 2014, pp. 331-333.

24. D. Q. M. Craig, The mechanisms of drug release from solid dispersions in water-soluble polymers, Int. J. Pharm. 231 (2002) 131-144; DOI: 10.1016/S0378-5173(01)00891-2.

25. T. L. Threlfall, Analysis of organic polymorphs. A review, Analyst 120 (1995) 2435-2460; DOI: 10. 1039/AN9952002435.

26. M. G. Issa and H. G. Ferraz, Intrinsic dissolution as a tool for evaluating drug solubility in accordance with the Biopharmaceutics Classification System, Dissol. Technol. 18 (2011) 6-13; DOI: 10. 14227/DT180311P6.

27. L. X. Yu, A. S. Carlin, G. L. Amidon and A. S. Hussain, Feasibility studies of utilizing disk intrinsic dissolution rate to classify drugs, Int. J. Pharm. 270 (2004) 221-227; DOI: 10.1016/j.ijpharm. 2003.10.016.

28. L. X. Yu, G. L. Amidon, J. E. Polli, H. Zhao, M. U. Mehta, D. P. Conner, V. P. Shah, L. J. Lesko, M. Chen, V. H. L. Lee and A. S. Hussain, Biopharmaceutics classification system: The scientific basis for biowaiver extensions, Pharm. Res. 19 (2002) 921-925; DOI: 10.1023/A:1016473601633.

29. P. Zakeri-Milani, M. Barzegar-Jalali, M. Azimi and H. Valizadeh, Biopharmaceutical classification of drugs using intrinsic dissolution rate (IDR) and rat intestinal permeability, Eur. J. Pharm. Biopharm. 73 (2009) 102-106; DOI: 10.1016/j.ejpb.2009.04.015.

30. M. El-Badry, M. A. Hassan, M. A. Ibrahim and H. Elsaghir, Performance of Poloxamer 407 as hydrophilic carrier on the binary mixtures with nimesulide, Farmacia 61 (2013) 1137-1150. 